\title{
Total Zinc in Zinc Battery Plates by EDTA Titration
}

\author{
Vernon L. Hammersley, Naval Surface Warfare Center, \\ Crane, IN 47522
}

\section{INTRODUCTION:}

At present, zinc battery plate electrodes are analyzed for zinc oxide, zinc chloride, zinc fluoride, zinc carbonate, zinc oxychloride, total zinc, zinc as the metal, and trace metals. A variety of methods are used to determine each of these components. The amount of zinc in each of the zinc compounds is determined by multiplying the percent of the compound by the ratio of the molecular weights of zinc to the zinc compound. This percent zinc is subtracted from percent total zinc and the operation is performed for every zinc compound determined. The remaining zinc value after these subtractions represents zinc as the metal. Zinc metal is the charged state on the anode. Percent total zinc is required in all these calculations.

The importance of these components cannot be overemphasized. The presence, or absence, of certain components in the zinc electrode can influence its behavior in a zinc-silver oxide primary battery. Passivation layers, tendency to dendritic growth, corrosion rates, voltage rise times, current density, porosity, surface area, electrochemical capacity, and other considerations make it imperative that the chemical composition of the zinc electrode be known.

The focus of this project was to evaluate the present method for total zinc and to develop a better method. The present method uses the disodium salt of ethylenediamine tetraacetic acid (EDTA) with Eriochrome Black $\mathrm{T}$ as the indicator. Basically, the use of EDTA to determine metals can be represented by the following formula:

$$
\mathrm{M}-\mathrm{In}+\text { EDTA }-\mathrm{M}-\mathrm{EDTA}+\mathrm{In}
$$

Where M-In represents the metal-indicator complex, M-EDTA represents the metal-EDTA complex and $I n$ is the free indicator. ${ }^{1}$

US Government work not protected by US copyright
The metal-indicator complex is less stable than the metal-EDTA complex so that as EDTA is added the metal is taken from the metal-indicator complex to form the more stable metal-EDTA complex. This is especially true with divalent ions such as zinc in basic or slightly acidic solutions. When all the metal is in the form of the metal-EDTA complex the free indicator remains. With Eriochrome Black $T$ the reaction can be represented by the following formula with color changes indicated.

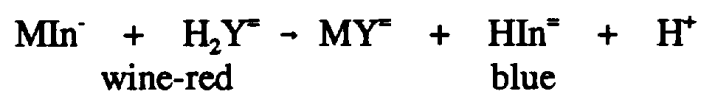

The $\mathrm{H}_{2} \mathrm{Y}^{=}$represents the ionized form of disodiumEDTA while $\mathrm{MY}^{2}$ is the metal-EDTA complex ion and $\mathrm{HIn}^{=}$is the ionized indicator. Note that hydrogen ion is formed in this reaction as well. ${ }^{2}$

\section{INITIAL CONSIDERATIONS:}

The approach taken for a new method was to remain with EDTA as the titrant, but change the indicator. The logic of changing the indicator was to see if a color change could be found that was sharper and easier to detect. With a sharper endpoint the analysis would be more accurate and precise. This was the goal for selecting another indicator.

The use of indicators dates back to at least $1881 .^{3}$ Detection of indicator color changes depends on the sensitivity of the human eye. An excellent literature source for the properties of the eye and its sensitivity in detecting color changes is found in the literature ${ }^{4}$ which stresses that no two person's eyes are alike. This fact governs the conduct of the analytical chemist in the laboratory. A conscientious worker will not do an analysis using someone else's standardized solution unless accuracy is not required. This is the case with 
Eriochrome Black $\mathrm{T}$ where a question arises as to whether the endpoint is deep blue yet or does it still have a grape color to it?

Important considerations when using EDTA for analysis are numerous. When determining a certain metal the presence of other interferring metals may be a problem. The reagents required for sample preparation may influence whether EDTA can be used or not. Also, the $\mathrm{pH}$ of the solution is very critical. Not only does EDTA use depend on $\mathrm{pH}$, but the indicator changes are governed by it as well. The stability of the indicator is important. One indicator may fade rapidly, while another may react with the metal in a way to render it unuseable. Other metals present in solution may eliminate certain indicators from consideration.

The use of Eriochrome Black $\mathrm{T}$ has been praised as an excellent choice for many metals including zinc. However, under the conditions of the battery plate analysis the endpoint is not sharp. After the zinc solution is buffered to $\mathrm{pH} 10$ with ammonium chloride/ammonium hydroxide, and addition of Eriochrome Black $\mathrm{T}$ dissolved in triethanolamine, it is titrated with $0.1 \mathrm{~N}$ EDTA. The color is initially wine-red and changes to a deep blue at the endpoint. Unfortunately, the change is not sharp. The color proceeds from wine-red, through shades of purple, to light blue, then deep blue.

Difficulty in detecting the Eriochrome Black $\mathrm{T}$ endpoint has resulted in total zinc values of more than $100 \%$ on occasions. Besides the difficulty in detecting the endpoint, another problem was noted during these analyses. The reaction of EDTA with zinc is not instanteous. If time is allowed between addition of each drop of EDTA near the endpoint, then some color change can be detected visually. The change is still not sharp, but values over $100 \%$ were not obtained.

A number of indicators suitable for use in the EDTA titration of zinc are reported in the literature. ${ }^{5}$ Consideration was given to several of these. Candidates were selected on the basis of type of color change, effective $\mathrm{pH}$ range, freedom from interference by other metals present in the battery plate material, stability, ease of preparing the indicator solution, and availability. Other important factors to take into account are the stability constants of the metal-indicator and metal-EDTA complexes and how they relate to each other. The relationship of these two complexes must be correct before the titration will work. ${ }^{6}$

Once the indicators were selected the approach was to use high purity zinc shot to check the precision and accuracy of the method with each indicator including Eriochrome Black T. The standardization of the EDTA was conducted using Eriochrome Black T. This value was used to compute the percent zinc using all indicators under consideration.

\section{EXPERIMENTAL:}

Indicators that were selected besides Eriochrome Black T were Xylenol Orange, PAN and a mixture of Eriochrome Black $\mathrm{T}$ and Methyl Orange. Murexide, although employed for zinc in certain applications, was not considered. Zinc forms an orange or yellow complex with Murexide, but the complex is said to be of low stability and would not be of value analytically.?

All indicators, except PAN, were mixed with sodium chloride. This provided an inert filler, and the indicator was more stable in this form than in a solution form. PAN, however, had to be made up in methanol solution because of its insolubility in water

Zinc shot was used for the standard. To observe the accuracy and precision, a total of at least ten titrations were performed using each indicator. Several statistical parameters were determined from these titrations. After the initial work with a zinc standard was completed a composite of battery plate material was prepared. At least ten titrations with each indicator followed by statistical calculations were performed on this sample as well.

\section{RESULTS AND DISCUSSION:}

Standard deviation and the mean indicate how consistent results are. In the table below $S$ is standard deviation, and $\mathrm{x}$ is the mean. Eriochrome 
Black T was run twice. Xylenol Orange was run twice, once with a pH 5.0 buffer and once with a $\mathrm{pH} 5.5$ buffer. The results in Table 1 were obtained on the zinc standard:

\begin{tabular}{|lrr|}
\hline \multicolumn{4}{|c|}{ Table 1 } & \\
Statistical Data - Zinc Standard Titration \\
Indicator & \multicolumn{1}{c|}{$\mathrm{S}$} & $\mathrm{x}$ \\
Eriochrome Black T \#1 & 0.38 & 100.09 \\
Eriochrome Black T \#2 & 0.22 & 100.23 \\
Black T + Methyl Orange & 0.18 & 100.21 \\
PAN, pH 5.0, NaOH & 0.38 & 98.79 \\
Xylenol Orange pH 5.0, NaOH & 0.22 & 99.74 \\
Xylenol Orange pH 5.5, $\mathrm{NH}_{4} \mathrm{OH}$ & 0.12 & 100.01 \\
PAN, pH 5.5, $\mathrm{NH}_{4} \mathrm{OH}$ & 0.36 & 98.68 \\
\hline
\end{tabular}

Variations in the two Eriochrome Black T runs show the inconsistencies encountered with this indicator. A correct and consistent judgement of the end point is difficult. The addition of methyl orange to Eriochrome Black $T$ improved the end point detection considerably. In this case the color change was from pink to green, an easier change to detect than the pink to deep blue.

The color change for PAN was pink to orange, a change that is difficult to detect as can be seen from the high standard deviation of 0.38 .

Xylenol Orange performed the best. Its color change was from pink to yellow. The more acidic buffer of $\mathrm{pH} 5.0$ required periodic addition of sodium hydroxide during the EDTA titration to prevent the $\mathrm{pH}$ from dropping below 5.0. The disodium EDTA has two hydrogen groups which gives it an acid character. The buffering capacity of the $\mathrm{pH} 5.5$ buffer was greater and required no $\mathrm{pH}$ adjustment during titration. Without the necessity of $\mathrm{pH}$ adjustment the more concentrated $\mathrm{pH} 5.5$ acetate buffer provided a stable $\mathrm{pH}$ environment for the titration. In an analytical method that is sensitive to $\mathrm{pH}$ this allowed more consistent results as evidenced by the lowest standard deviation of 0.12 and mean value of 100.01 .

A composite sample of zinc battery plate material was prepared by mixing anode material from two batteries together, grinding, then passing through a 100 mesh sieve. Prior to using each of the four indicators with plate material the EDTA was standardized with Eriochrome Black $T$ and the zinc standard. As can be seen from the results in Table 2, the standard deviation was greater than for the zinc standard for all indicators. During neutralization with sodium hydroxide in the Xylenol Orange procedure, the precipitate that was encountered did not redissolve during addition of the acetate buffer as was the case with the zinc standard. The endpoint was difficult to determine accurately and a higher standard deviation with Xylenol Orange was encountered. When ammonium hydroxide was used to neutralize the nitric acid prior to addition of the buffer no precipitate was formed. Zinc forms a complex ion with ammonia which remains in solution. Results tabulated show a lower standard deviation for Xylenol Orange.

\section{Table 2}

Statistical Data - Anode Material Titration

\begin{tabular}{lll} 
Indicator & $\mathrm{S}$ & $\mathbf{x}$ \\
Eriochrome Black T & 0.40 & 95.39 \\
Black T + Methyl Orange & 0.43 & 95.26 \\
Xylenol Orange, pH 5.5, NaOH & 0.50 & 94.92 \\
Xylenol Orange, pH 5.5, $\mathrm{NH}_{4} \mathrm{OH}$ & 0.33 & 95.38 \\
PAN, pH 5.5, $\mathrm{NH}_{4} \mathrm{OH}$ & 0.46 & 93.49 \\
\hline
\end{tabular}

A higher standard deviation for zinc battery plate material as compared to standard zinc shot comes as no surprise. Zinc battery plate material contains mercury, copper, iron, and a variety of zinc compounds that were previously mentioned. Mercury is purposely added to reduce the corrosion rate in $\mathrm{KOH}^{8}$ Copper and iron often find their way into zinc plates at some point during plate manufacturing. Zinc oxide and zinc carbonate can form through the exposure of zinc metal to oxygen, carbon dioxide and moisture. The chlorides and fluoride may be the result of contamination during the forming and washing processes. Given that all these components could be in a homogeneous mixture with zinc metal in the plate material, the 
analytical results are not expected to be as precise as with a zinc standard. The effects of most of these components in an EDTA titration are not known. Considering all the components that could be present, the standard deviation was surprisingly small.

A number of other statistical calculations may be performed on the titration data, but standard deviation and mean serve to show the precision and accuracy of these methods.

\section{CONCLUSIONS}

There are advantages and disadvantages to every method of analysis. The use of Eriochrome Black T or Xylenol Orange in the titration of zinc with EDTA is no exception. The advantage of using Eriochrome Black $T$ is that the solution is basic with ammonium hydroxide and the ammonium chloride/ammonium hydroxide $\mathrm{pH} 10$ buffer is strong enough that monitoring the solution with a $\mathrm{pH}$ meter during titration is unnecessary. The disadvantage is the difficulty in perceiving the endpoint consistently. The advantage to using Xylenol Orange is the improved precision in visual detection of the endpoint. The disadvantage is that the neutralization must be monitored with a pH meter and stopped around $\mathrm{pH} 5.5$ or 6.0. A higher solution $\mathrm{pH}$ will not be buffered to $\mathrm{pH} 5.5$, because the acetate buffer is not strong enough.

The use of Xylenol Orange in preference to Eriochrome Black T is recommended. The EDTA solution can be standardized with Xylenol Orange prior to analyzing battery plate material. The tabulated data show significant improvement in precision with Xylenol Orange over the other indicators tested. This is true for both zinc shot standard and battery plate material.

\section{REFERENCES:}

1. Frank J. Welcher, The Analytical Uses of Ethylenediamine Tetraacetic Acid, D. Van Nostrand Co., Inc., page 32, (1958).

2. ibid., page 34 .

3. Aaron J. Ihde, The Development of Modern Chemistry, Dover Publications Inc., page $560,(1984)$.
4. M. G. Mellon, Analytical Absorption Spectroscopy, John Wiley \& Sons, pp 515-600, (1950).

5. A. J. Barnard, Jr., W. C. Broad, and H. Flaschka, Chemist-Analyst, Vol. 45, No. 4, Dec. 1956; Vol. 46, No. 1, Mar. 1957; Vol. 46, No. 2, June 1957.

6. A. J. Barnard, Jr., W. C. Broad, and H. Flaschka, Chemist-Analyst, Vol. 45, No. 4, pp 8889, Dec. 1956.

7. Frank J. Welcher, The Analytical Uses of Ethylenediamine Tetraacetic Acid, D. Van Nostrand Co., Inc., page 62, (1958).

8. Arthur Fleischer and John J. Lander, ZincSilver Oxide Batteries, John Wiley \& Sons, page 87, (1971). 